\title{
Analysis of narrowband power line communication channels for advanced metering infrastructure
}

\author{
José Antonio Cortés ${ }^{*}$, Alfredo Sanz, Pedro Estopiñán and José Ignacio García
}

\begin{abstract}
This paper analyzes the characteristics of narrowband power line communication (NB-PLC) channels and assesses their performance when used for advanced metering infrastructure (AMI) communications. This medium has been traditionally considered too hostile. However, the research activities carried out in the last decade have shown that it is a suitable technology for a large number of applications. This work provides a statistical characterization of NB-PLC channels in the CENELEC-A band. The presented results have been obtained from a set of 106 links measured in urban, suburban, and rural scenarios. The study covers the input impedance of the power line network, the channel response and the noise. The analysis of the channel response examines the delay spread, the coherence bandwidth, and the attenuation, while the assessment of the noise considers both its spectral and temporal characteristics. Since low voltage (LV) distribution networks consists of several conductors, they can be simultaneously used to set up multiple-input multiple-output (MIMO) communication links. This paper investigates the correlation between the MIMO streams. The bit rates that can be attained both in the single-input single-output (SISO) and in the MIMO cases are estimated and discussed.
\end{abstract}

Keywords: Power line communications; Narrowband; Smart Grid; Advanced metering infrastructure

\section{Introduction}

The conventional paradigm of electricity networks 'generate what is consumed' is shifting towards the new 'consume what is produced' [1,2]. This change is motivated by facts like the increased use of renewable sources, which have a much more decentralized structure than conventional ones and whose generating capacity is subject to unpredictable factors, and by new consumption patterns like electric vehicles charging, which complicate the demand forecasting.

The traditional electricity network must evolve into the so-called smart grid to support this change. Advanced metering infrastructure (AMI) is considered a constituent part of the Smart Grid. It enables applications such as automatic meter reading (AMR), demand side response, and distribution automation [1,3]. AMI requires bidirectional communication links between the medium voltage

*Correspondence: jose_antonio.cortes@atmel.com

Atmel Spain, Torre C2, Polígono Puerta Norte, A-23 Zaragoza, Spain to low voltage (MV/LV) transformer stations and the costumers, usually known as the last mile. Recent studies suggest that power line communications (PLC) is the most cost-effective technology for ARM [4]. In addition, it easily enables power quality measurements and distribution automation functions; it gives utilities full control of the communication network and seems to be the most appropriate technology for the communication between the on-board charging system of electric vehicles and the grid [5].

PLC technology can be classified in terms of the employed bandwidth into narrowband (NB) and broadband $(\mathrm{BB})[5,6]$. Data rates estimated for last-mile AMI applications suggest that they can be delivered by NBPLC in a more inexpensive way than with BB-PLC $[7,8]$. Examples of suitable systems for this end are the ones defined in the ITU-T Recommendations G.9902 (known as G.hnem), G.9903 and G.9904, and the IEEE P1901.2 [9-12]. ITU-T G.9903 and G.9904 are based on the industry specifications G3-PLC and Powerline Intelligent Metering Evolution (PRIME), respectively.

\section{望 Springer}

(c) 2015 Cortés et al.; licensee Springer. This is an Open Access article distributed under the terms of the Creative Commons Attribution License (http://creativecommons.org/licenses/by/4.0), which permits unrestricted use, distribution, and reproduction in any medium, provided the original work is properly credited. 
Nowadays, the CENELEC-A band ( 3 to $95 \mathrm{kHz}$ ) is the most widespread one in NB-PLC [13]. However, the quantitative knowledge of the channel in this frequency range is still imprecise [14]. This is clearly reflected by the significant differences among the physical layer parameters of the latest NB-PLC systems [5]. Recent noise measurements and models incorporated into the IEEE P1901.2 have provided much insight into the noise features in the frequency band above $100 \mathrm{kHz}$ [15]. Nevertheless, their suitability for the CENELEC-A band has not been assessed. The same uncertainty applies to the 'fading modeling method' stated in the IEEE P1901.2 for the channel response. It results from a particularization of the wellknown model proposed in [16], but the appropriateness of the selected parameters to generate responses in the CENELEC-A band has not been evaluated. Similarly, measurements performed in selected scenarios have provided much information about the qualitative features of the channel response $[15,17,18]$, but the absence of a statistical knowledge leads to a large uncertainty in the expected performance of NB-PLC.

LV distribution networks consist of several conductors which can be simultaneously used to set up multipleinput multiple-output (MIMO) communication links. This strategy is being successfully employed in wireless communications and in indoor BB-PLC [19], where the phase, neutral, and protective earth conductors are used for the MIMO. However, there are almost no available works on NB-PLC MIMO, and they are limited to explore indoor NB-PLC channels [20] or to plain tests using existing single phase PRIME devices [21].

In this context we make three main contributions:

- We provide a statistical analysis of the channel characteristics in the CENELEC-A band. These results are compared to the ones obtained with the more recent channel model for this band, proposed in the IEEE P1901.2 standard.

- We analyze the correlation between the channels of the $3 \times 3$ MIMO links that could be established by injecting and receiving the communication signal between each of the phases and the neutral conductor.

- We estimate the data rates that could be achieved both in single-input single-output (SISO) and MIMO communications. These results will be useful to clarify whether there is a need for using BB-PLC in the last mile of AMIs.

The rest of the paper is organized as follows. Section 2 provides a brief description of the employed measurement setup and signal processing algorithms. Sections 3 and 4 are devoted to the characterization of the input impedance of the power line network and its channel response, respectively. Section 5 is the noise counterpart of Section 4. Based on the presented characterization, Section 6 provides an estimate of the achievable performance. Finally, Section 7 summarizes the main conclusions.

\section{Measurement methodology}

\subsection{Measurement setup}

The measurement setup consists of two equal signal generation and data acquisition systems, as shown in Figure 1. They are controlled by a laptop, which also stores the acquired signals. Measurements are differentially performed from the three phases to the neutral conductor. The signal generation system (SGS) comprises a signal generation board (SGB), a high power amplifier (implemented using a parallel structure), and a coupling circuit. The SGS has very low output impedance, its real part is about $220 \mathrm{~m} \Omega$, and is able to deliver $116 \mathrm{~dB} \mu \mathrm{V}$ over $2 \Omega$ load. The data acquisition system (DAS) has a coupling circuit, a band pass filter (BPF), and a 16-bits analog to digital converter (ADC) with a sampling frequency of $f_{s}=1 \mathrm{Msamples} / \mathrm{s}$. The use of a high-resolution ADC and a large oversampling factor avoids the need for an amplifier. The reason is that the power spectral density (PSD) of the quantization noise is $-33.14 \mathrm{~dB} \mu \mathrm{V} / \sqrt{\mathrm{Hz}}$, which is between $42 \mathrm{~dB}$ (at low frequencies) and $28 \mathrm{~dB}$ (at high frequencies) lower than the PSD of the weakest measured noise, as it will be shown in Section 5. The input impedance of the DAS is about $1.2 \mathrm{k} \Omega$. Hence, the loading effect is negligible, since the largest input impedance values of the power line network are on the order of tenths of $\Omega$.

Estimates of the channel response are computed from the input and output signals $v_{T}(t)$ and $v_{R}(t)$ shown in Figure 1. Therefore, the attenuation due to both coupling circuits (about $1 \mathrm{~dB}$ in the passband) and the coupling loss between the SGS and the power line network are measured as part of the channel. The latter effect could be compensated, since the input impedance of the channels has also been measured. In fact, this must be done when the results are to be used in a channel emulator which separately models both magnitudes, channel response and input impedance [17]. However, the characterization accomplished in this work is intended for assessing the performance of actual communication systems. Hence, all the effects that are present in a real situation have to be taken into account, including impedance mismatch.

The characterization of the $3 \times 3$ MIMO links is done using the sequential measurements of the nine SISO channels accomplished with the aforementioned setup. Since the input phases not involved in the SISO channel that is being measured are left open circuit, this does not exactly model the actual situation in which these phases would be loaded with the impedance of the MIMO transmitter. 


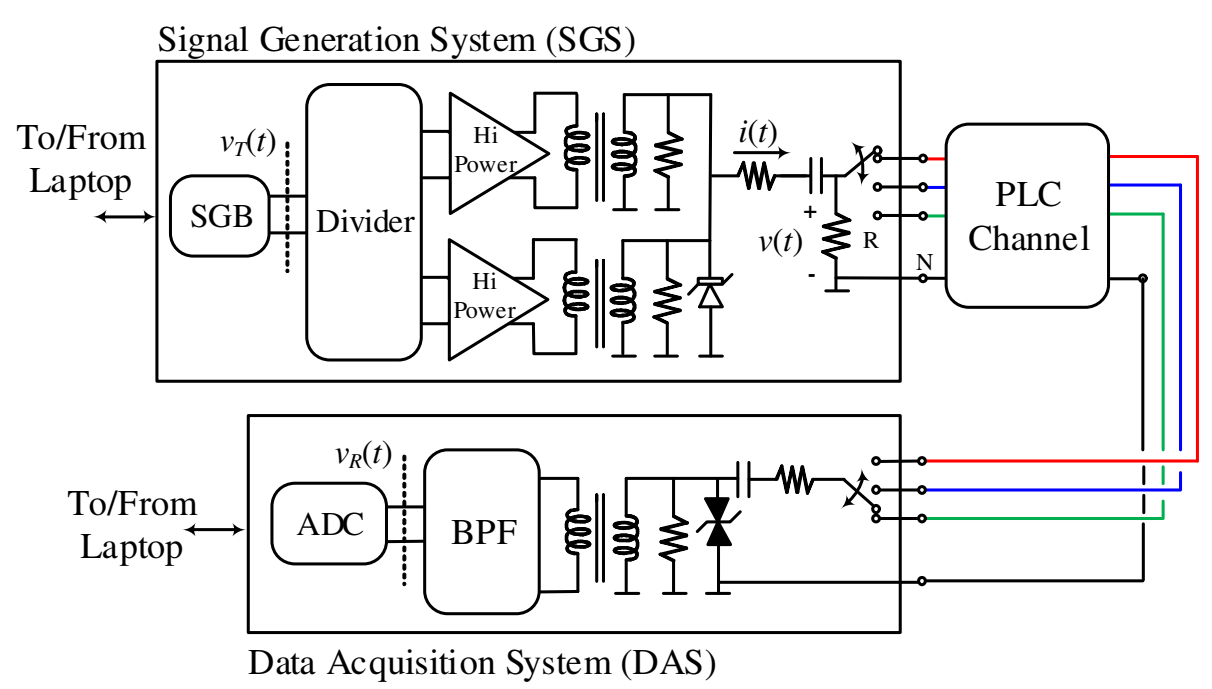

Figure 1 Simplified scheme of the measurement setup.

However, this does not limit the validity of the results since the influence of the impedance connected to the unused phases has proven to be negligible. Simulations using multiconductor transmission line (MTL) theory have shown that the response of the MIMO streams and their spatial correlation is essentially determined by the cable characteristics, by the topology of the underlying LV line, and by the loads connected to the same phase.

Measurements have been carried out in nine networks in the Center and North West of Spain. Table 1 summarizes the information of each site. A total of 106 links have been measured in three environments: rural (27), suburban (29), and urban (50). Each of them is characterized as a $3 \times 3$ MIMO. Hence, the analysis involves 954 channel responses. Measurements are always performed in the LV part of the network, generally between the LV busbar of the $\mathrm{MV} / \mathrm{LV}$ transformer and the meter position. However, measurements are performed at intermediate locations in cases like pole-mounted transformers. As shown in Table 1, cables are generally deployed underground (UG) in the urban scenario, overhead $(\mathrm{OH})$ in the rural one, and a mix of both in the semiurban scenario. In rural areas, all customers are connected to a single LV line, while in urban ones, the number of lines deployed from the transformer station is much larger. Table 1 also indicates the linear distance between the transformer station and the closest and the farthest meters.

\subsection{Measurements processing}

The noise signal in the $j$ th port, $n^{j}(t)$, is registered during $C=26$ European mains cycles. Since NB-PLC noise has cyclostationary nature, magnitudes employed for its characterization must be computed synchronously with the mains. To this end, the jitter of the mains signal in the $c$ th cycle, $\tau^{j}(c)$, must be taken into account. Hence, each mains cycle is divided into $L$ intervals with $N_{L}=\frac{f_{s}}{f_{o} L}$

Table 1 Description of the measured scenarios

\begin{tabular}{|c|c|c|c|c|c|c|c|}
\hline Site & Scenario & Cabling & $\begin{array}{l}\text { Number of } \\
\text { LV lines }\end{array}$ & $\begin{array}{l}\text { Number of } \\
\text { customers }\end{array}$ & $\begin{array}{l}\text { Min. } \\
\text { dist. (m) }\end{array}$ & $\begin{array}{l}\text { Max. } \\
\text { dist. (m) }\end{array}$ & $\begin{array}{l}\text { Number of } \\
\text { MIMO links }\end{array}$ \\
\hline 1 & Rural & $\mathrm{OH}$ & 2 & 126 & 6 & 427 & 10 \\
\hline 2 & Rural & $\mathrm{OH}$ & 1 & 105 & 14 & 359 & 6 \\
\hline 3 & Rural & $\mathrm{OH}$ & 1 & 66 & 10 & 710 & 5 \\
\hline 4 & Rural & $\mathrm{OH}$ & 1 & 67 & 12 & 1063 & 6 \\
\hline 5 & Semiurban & $\mathrm{OH}$ & 2 & 124 & 16 & 615 & 10 \\
\hline 6 & Semiurban & UG & 4 & 132 & 5 & 163 & 6 \\
\hline 7 & Semiurban & $\mathrm{OH}$ & 2 & 183 & 34 & 423 & 13 \\
\hline 8 & Urban & $\mathrm{OH} \& U \mathrm{UG}$ & 24 & 737 & 4 & 340 & 36 \\
\hline 9 & Urban & UG & 12 & 329 & 19 & 65 & 14 \\
\hline
\end{tabular}


samples, where $f_{o}$ denotes the frequency of the mains signal. The nominal and actual lengths of the $c$ th mains cycle are $N_{C}=f_{s} / f_{o}$ and $N_{C}+\tau^{j}(c)$ samples, respectively. The noise captured in the $j$ th port during the $\ell$ th interval of the $c$ th cycle can then be written as:

$$
n_{c, \ell}^{j}(n)=n^{j}\left(n+c N_{c}+\sum_{i=0}^{c} \tau^{j}(i)+\ell N_{L}\right),
$$

with $0 \leq n \leq N_{L}-1$.

This framework is employed to assess the spectral and temporal characteristics of the noise. In this paper, the former is accomplished in terms of the PSD, which is estimated by means of the periodogram:

$$
P_{c}^{j}(\ell, k)=\frac{1}{U N_{L}}\left|\sum_{n=0}^{N_{L}-1} w(n) n_{c, \ell}^{j}(n) e^{-j \frac{2 \pi}{N_{L}} k n}\right|^{2},
$$

where $w(n)$ is a Hanning window of $N_{L}$ samples and $U$ is the normalization factor that removes the estimation bias [22]. The cyclostationary and periodic components of the noise have a frequency multiple of the mains. Hence, an estimate of the frequency sampled version of the PSD can be obtained by performing an averaging of the periodograms in (2) with $L=2$ :

$$
\widehat{S}_{N^{j}}(k)=\left.\widehat{S}_{N^{j}}(f)\right|_{f=k \frac{f_{s}}{N_{L}}}=\frac{1}{C L} \sum_{c=0}^{C-1} \sum_{\ell=1}^{L} P_{c}^{j}(\ell, k) .
$$

The employed value of $L$ yields a frequency resolution of $100 \mathrm{~Hz}$.

In order to characterize the noise variation, each mains cycle has now been divided into $L=80$, and the average value of the energy (along multiple mains cycles) in these intervals is estimated as:

$$
E^{j}(\ell)=\frac{1}{C} \sum_{c=1}^{C} \sum_{n=0}^{N_{L}-1}\left|n_{c, \ell}^{j}(n)\right|^{2} .
$$

The selected value of $L$ leads to a time resolution of 250 $\mu \mathrm{s}$.

Channel response measurements are obtained by transmitting an orthogonal frequency division multiplexing (OFDM)-like sounding signal generated using a 2048 point DFT. Its lengths also equals $C=26$ European mains cycles. An estimate of the frequency response is then obtained by averaging the least squares (LS) estimations obtained from each symbol. The moderate length of the acquired signal has obliged to accomplish an asynchronous averaging (with respect to the mains) of the LS estimates in order to achieve a reasonable signalto-noise ratio (SNR). This provides an estimate of the average channel response in the frequency range 40 to 91 $\mathrm{kHz}$, veiling possible periodic variations in the channel response [23].
The input impedance of the power line channel is estimated during the transmission of the OFDM signal used for channel sounding. The current and voltage signals $i(t)$ and $v(t)$ shown in Figure 1 have been employed for this purpose, following a similar approach to the one in [24]. To this end, the resistance $R$ is fixed to a much larger value than the one of the PLC grid. For the sake of clarity, the circuits used for conditioning and digitizing $i(t)$ and $v(t)$ are not shown.

\section{Impedance characterization}

The input impedance of the power line network in the considered band is frequency selective. Its magnitude generally increases with frequency, reaching maximum values of tenths of $\Omega$. Illustrative shapes can be found in $[18,24]$.

Figure 2 depicts the cumulative distribution function (CDF) of the frequency-averaged magnitude of the impedance values measured in each scenario. As seen, the median values (50\% probability) are always below $10 \Omega$. Moreover, in the semiurban and urban scenarios, they are even below $5 \Omega$. These scenarios exhibit lower impedance values, with respect to the rural one, mainly because of the loading effect caused by the customers located in the same metering room.

The magnitudes shown in Figure 2 pose a twofold problem in the design of NB-PLC systems. The first one is the difficulty of injecting signal levels of up to $5 \mathrm{~V}$, as the ones allowed by the EN 50065-1 [13], into such low impedance values. The second one is the minimization of the coupling loss from the transmitter to the PLC network. To this end, the most desirable situation is to make the output impedance of the transmitter negligible with respect to the input impedance of the PLC grid. However, this obliges to make an output impedance on the order of a few $\mathrm{m} \Omega$, which is in the range of the resistance of some printed circuit board traces or the transformers wiring. On the other hand, conjugate impedance matching is also technologically difficult because of the aforementioned frequency-selective behavior of the impedance and its large variation between locations.

\section{Channel response characterization}

NB-PLC channel responses are frequency selective. Figure 3 depicts the channel response of six actual channels, two per measured scenario. One of the channels can be assumed to have an 'average' response, and the other is among the worst channels measured in the corresponding scenario. The frequency selectivity is caused by reflections at discontinuities, which lead to a multipath propagation phenomenon and by the loading effect of the in-home network. However, in Europe, the latter seems to be more important in the CENELEC-A band. The reason is that links are usually too short for the conductors to behave as transmission lines. As a rule of thumb, this occurs when 


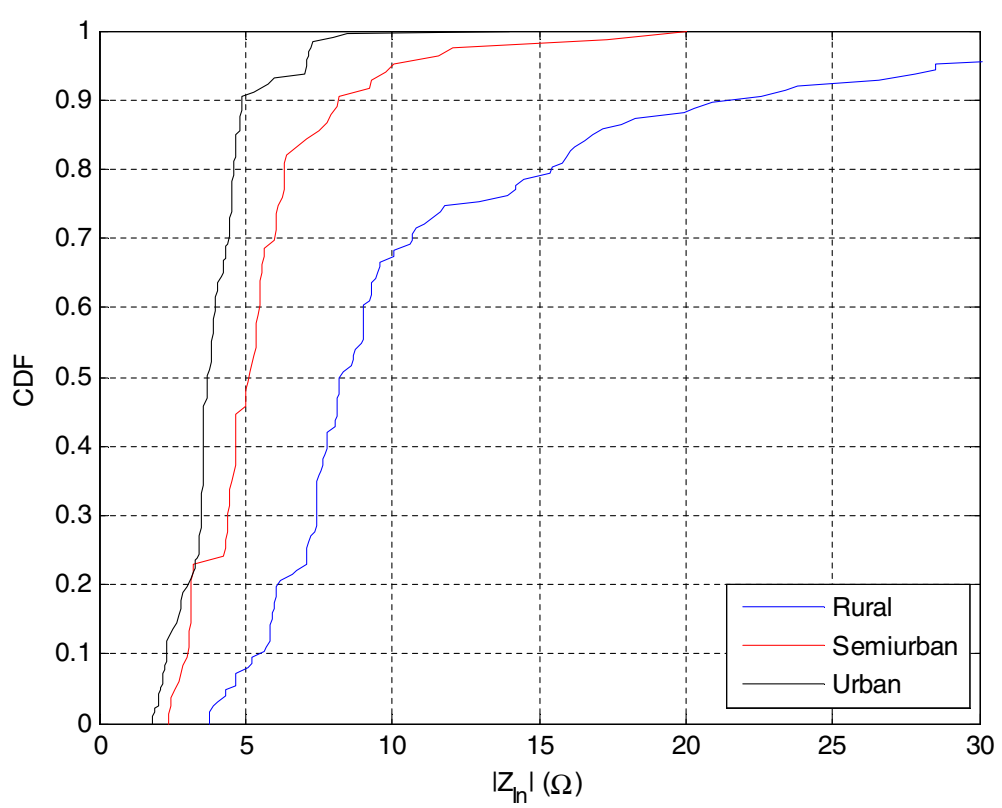

Figure 2 CDF of the frequency-averaged magnitude of the input impedance.

the wavelength of the transmitted signal is, at least, ten times larger than the involved distances. In the employed frequency band (40 to $91 \mathrm{kHz}$ ), this translates into 5.25 and $2.33 \mathrm{~km}$, respectively, which are much longer than the distances usually involved in European grids [25].

\subsection{Statistical analysis}

The coherence bandwidth and the delay spread are the most widespread parameters used to characterize the frequency selectivity of a channel response and its timedomain counterpart, the time dispersion. In this paper, the former is computed as the frequency separation for which the spaced-frequency correlation function falls down to 0.9 [26]. Both magnitudes have a plain relation to the parameters of OFDM communication systems, like the ones currently used in NB-PLC. In particular, distortion in the OFDM signal is avoided if the cyclic prefix is larger than the length of the channel impulse response.

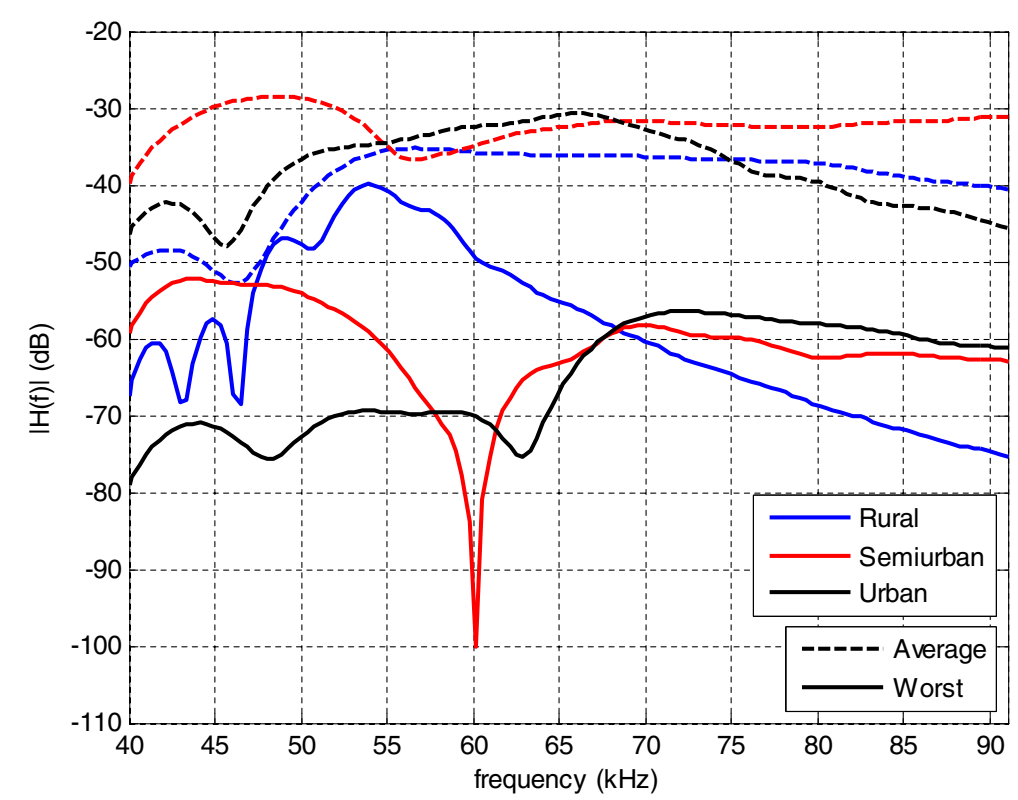

Figure 3 Attenuation profiles of example channels. 
Table 2 System parameters of NB-PLC systems standardized by the ITU-T

\begin{tabular}{llll}
\hline Parameter & $\begin{array}{l}\text { G.9902 } \\
\text { (G.hnem) }\end{array}$ & $\begin{array}{l}\text { G.9903 } \\
\text { (G3-PLC) }\end{array}$ & $\begin{array}{l}\text { G.9904 } \\
\text { (PRIME) }\end{array}$ \\
\hline Cyclic prefix length $(\mu \mathrm{s})$ & $60 / 120$ & 55 & 120 \\
Intercarrier spacing $(\mathrm{Hz})$ & 1562.5 & 1562.5 & 480 \\
FFT window length $(\mathrm{ms})$ & 0.640 & 0.640 & 2048 \\
Max bit rate (kbit/s) & 101.3 & 55.5 & 64.3 \\
\hline
\end{tabular}

Nevertheless, since increasing the cyclic prefix decreases the symbol rate, its optimum value is usually shorter than the channel impulse response length [27]. The delay spread is a root mean squared (rms) measure of the latter. Hence, the cyclic prefix length should generally be several times larger than the delay spread.

When an insufficient cyclic prefix is used, the magnitude of the distortion increases with the ratio of the carrier bandwidth to the coherence bandwidth of the channel. Hence, the coherence bandwidth can be also used to design the intercarrier spacing of an OFDM system. In order to assess the suitability of the parameters employed in the NB-PLC systems standardized by the ITU-T, their cyclic prefix, intercarrier spacing, FFT window length (i.e., symbol length excluding the cyclic prefix), and maximum bit rate are shown in Table 2. The bit rate indicated for PRIME corresponds to convolutionally coded transmissions. Larger bit rates can be achieved with uncoded transmissions.

Figure 4 depicts the CDF of the delay spread values corresponding to the channels measured in each scenario.
In order to assess the suitability of the 'fading modeling method' proposed in the IEEE P1901.2 standard, the CDF computed from 300 channels generated according to this model is also drawn. As seen, rural channels have larger delay spread values than urban and semiurban. This is likely due to the attenuation of the cables, which has a lowpass behavior. It can be also noticed that measured values are larger than modeled ones. In fact, the median value of the semiurban channels is about $45 \%$ larger than the median of the modeled ones. This figure rises up to $82 \%$ in the rural scenario. Conversely, measured channels have lower coherence bandwidth than modeled ones, as shown in Figure 5, where the CDF of the coherence bandwidth and the average attenuation is depicted. The comparison of the delay spread and the coherence bandwidth with the parameters shown in Table 2 reveals that all the systems have intercarrier spacings lower than the measured channel coherence bandwidth, although the value in the G.hnem/G3-PLC systems is about three times larger than in PRIME ${ }^{\mathrm{a}}$. Regarding the cyclic prefix, it seems that the value used in PRIME might be too large for the majority of channels, while the G3-PLC one seems to be too small for the worst channels. In terms of performance, G.hnem seems to provide the most appropriate solution.

Regarding the average attenuation of the measured and modeled channels, Figure 5 shows that semiurban channels experience lower attenuation than rural and urban ones. This might be due to the fact that semiurban scenarios use to involve shorter distances than rural ones and less derivations than urban environments. As seen, the spread of the attenuation in the modeled channels (around

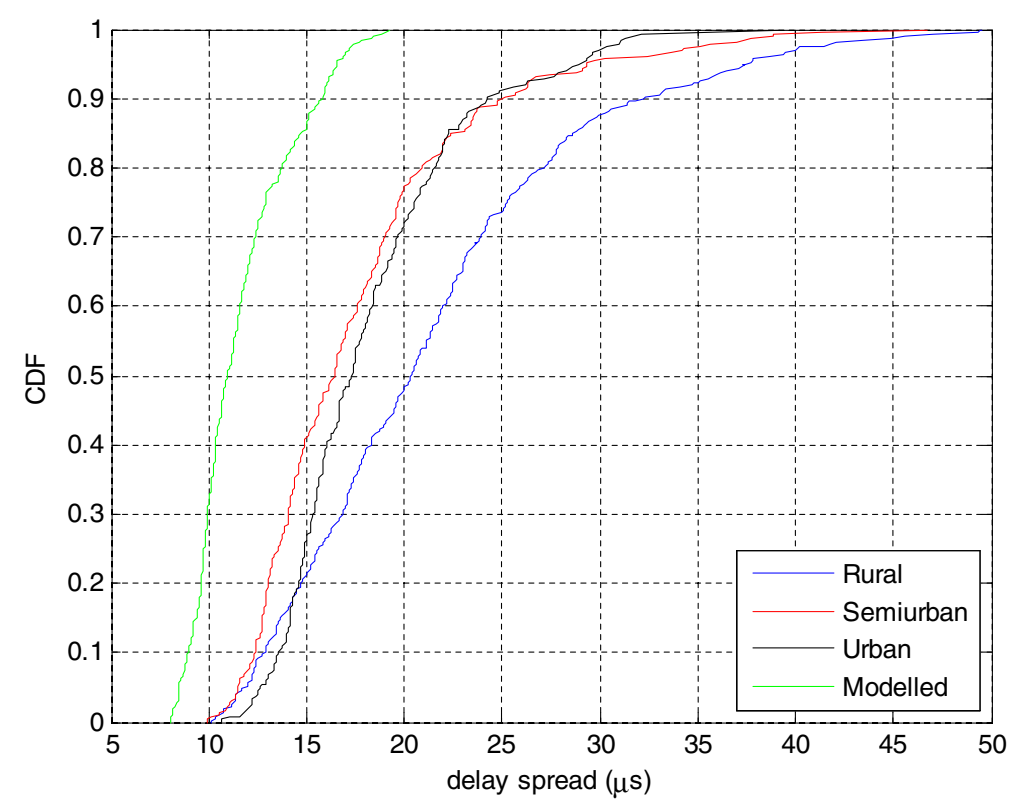

Figure $4 \mathrm{CDF}$ of the delay spread of the measured and modeled channels. 


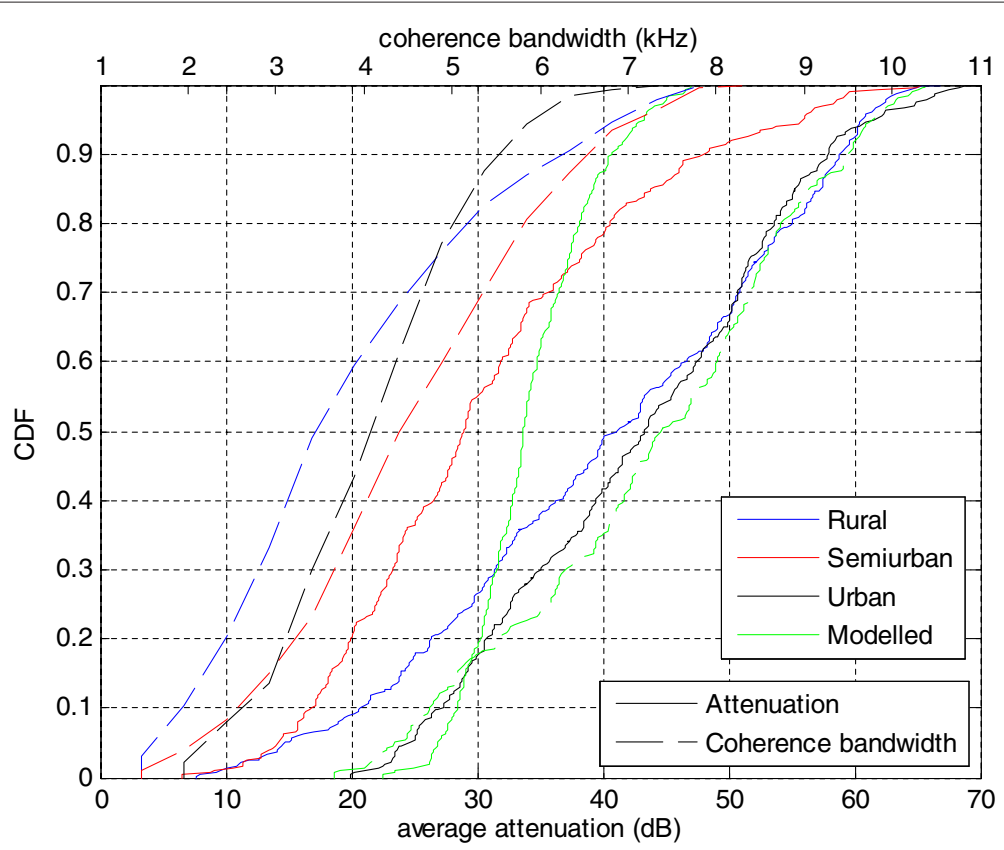

Figure 5 CDF of the average attenuation and the coherence bandwidth of the measured and modeled channels.

$25 \mathrm{~dB}$ ) is much smaller than in the measured ones. This can also be observed in Figure 6, where a scatter plot of the attenuation vs. the delay spread is drawn. As shown, the dispersion of the values in the measured channels is much larger than that in the modeled ones. It is also interesting to highlight that there is no clear correlation between attenuation and delay spread, as it happens in indoor
BB-PLC channels. This might be due to the reduced influence that the multipath propagation phenomenon has in NB-PLC, where derivations cause a much flatter power loss than in BB-PLC.

The presented results indicate that the parameters of the 'fading modeling method' are unsuitable for the CENELEC-A band, although they might be appropriate

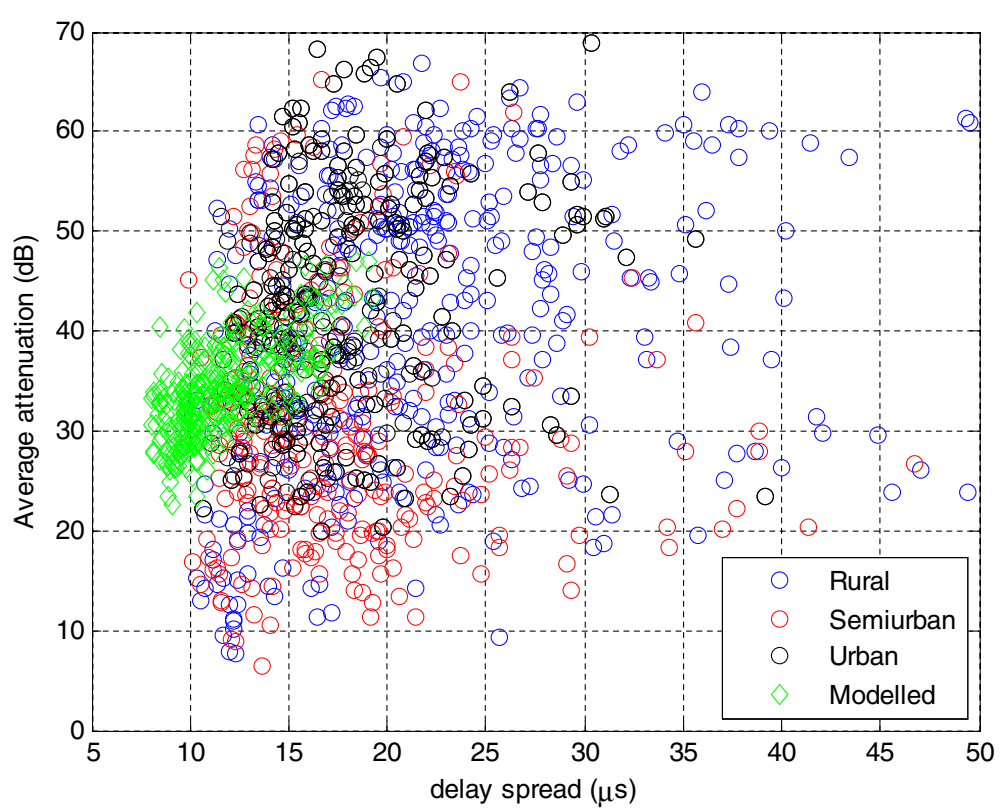

Figure 6 Relation between the average attenuation and the delay spread of the measured and modeled channels. 
for the 154.69 to $487.5 \mathrm{kHz}$ range, where the channel response is known to have better transmission characteristics. This band is defined in the IEEE P1901.2 for transmissions in the Federal Communications Commission (FCC) and the Association of Radio Industries and Businesses (ARIB) bands.

The characterization of the $3 \times 3$ MIMO links is accomplished by using the channel matrix at each frequency point, $\mathbf{H}$, where the frequency index is omitted for the sake of clarity. It is defined as:

$$
\mathbf{H}=\left(\begin{array}{lll}
H^{11} & H^{12} & H^{13} \\
H^{21} & H^{22} & H^{23} \\
H^{31} & H^{32} & H^{33}
\end{array}\right),
$$

where $H^{i j}$ denotes the frequency response of the channel between input port $i$ and output port $j$ at the corresponding frequency. The singular value decomposition (SVD) of $\mathbf{H}$ can be expressed as:

$$
\mathbf{H}=\mathbf{U D V}^{H} \text {, }
$$

where $\mathbf{U}$ and $\mathbf{V}$ are unitary matrices and $\mathbf{D}$ is a diagonal matrix whose values are the singular values, $\sigma_{i}$, and $(\cdot)^{H}$ denotes the Hermitian operator. The singular values are related to the eigenvalues of $\mathbf{H H}^{H}, \lambda_{i}$, as $\sigma_{i}=\sqrt{\lambda_{i}}$.

Hence, the SVD decomposes the MIMO channel into a set of orthogonal SISO channels, or streams, with amplitude $\sigma_{i}$. The ratio of the singular values can be used as a measure of the correlation between the constituent channels of the MIMO. If fact, the ratio of the maximum to the minimum singular value is the condition number of the matrix $\mathbf{H}$, denoted by $\kappa=\max \left(\sigma_{i}\right) / \min \left(\sigma_{i}\right)$. When the constituent channels of the MIMO are perfectly correlated, all singular values except one will be zero and the ratio is infinite. On the other side, when channels are absolutely uncorrelated, all the singular values have the same value and its ratio equals one.

Figure 7 depicts the values of $\kappa(\mathrm{dB})$ for the measured MIMO channels at each frequency point, computed as $20 \log _{10}(\kappa)$. As seen, it has a quite homogeneous behavior along the considered band in most channels. In addition, it can be observed that most values are larger than $10 \mathrm{~dB}$, which indicates that at least one of the MIMO stream is highly correlated with the others. Figure 8 corroborates this end. It shows the relevant part of the CDF of the singular values corresponding to the three MIMO streams and to the SISO channels. As seen, the singular values of the third stream are about $15 \mathrm{~dB}$ below the ones of the first stream. The median values of the first stream are between 4 and $6 \mathrm{~dB}$ larger than those of the SISO. Similarly, the median values of the second stream are between 1 and $4 \mathrm{~dB}$ lower than the SISO ones. The lowest singular values of the second and third MIMO streams, with respect to the SISO ones, occur in the rural environment. Hence, MIMO gains are expected to be lower here than in the other scenarios.

\section{Noise characterization}

Noise in NB-PLC is caused by the electrical devices connected to the power grid and to external signals coupled via radiation or via conduction. It is composed of three main components: background noise, narrowband interference (NBI), and impulsive noise. The former is

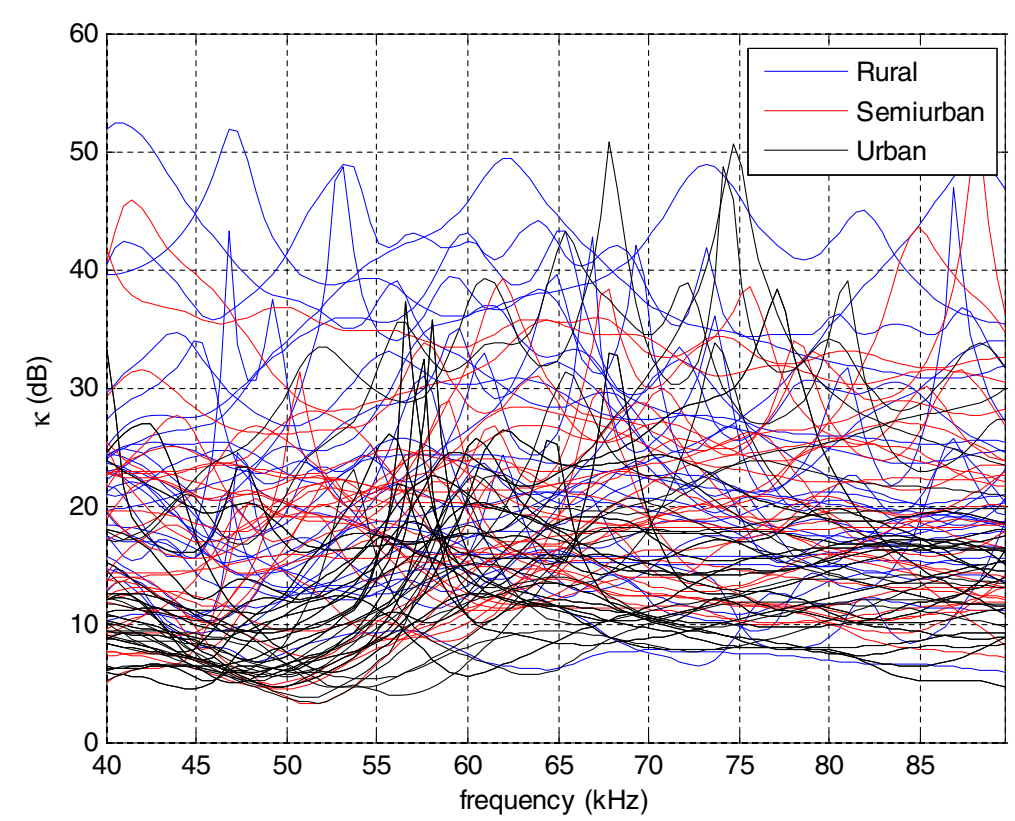

Figure 7 Ratio of the maximum to the minimum singular values. 


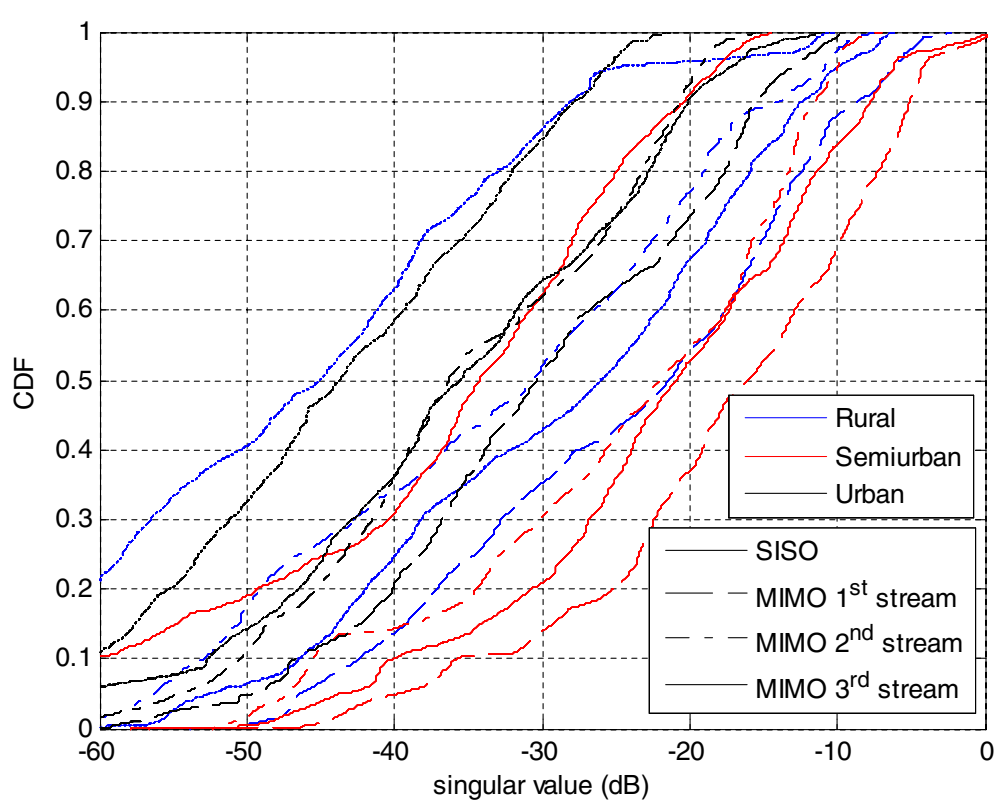

Figure $8 \mathrm{CDF}$ of the singular values.

generally cyclostationary with a frequency multiple of the mains cycle. The latter consists of sporadic (aperiodic) impulses as well as components that are synchronous to the mains [15]. Figure 9 depicts the spectrogram and the waveform of a measured noise register. The periodic impulsive term is clearly observable in both plots. In addition, it shows three high-level NBI around 59, 62, and 73 $\mathrm{kHz}$ and a sporadic impulse at around $35 \mathrm{~ms}$.

\subsection{Statistical analysis}

The first magnitude used to characterize the noise is the PSD, synchronously computed as indicated in Section 2.

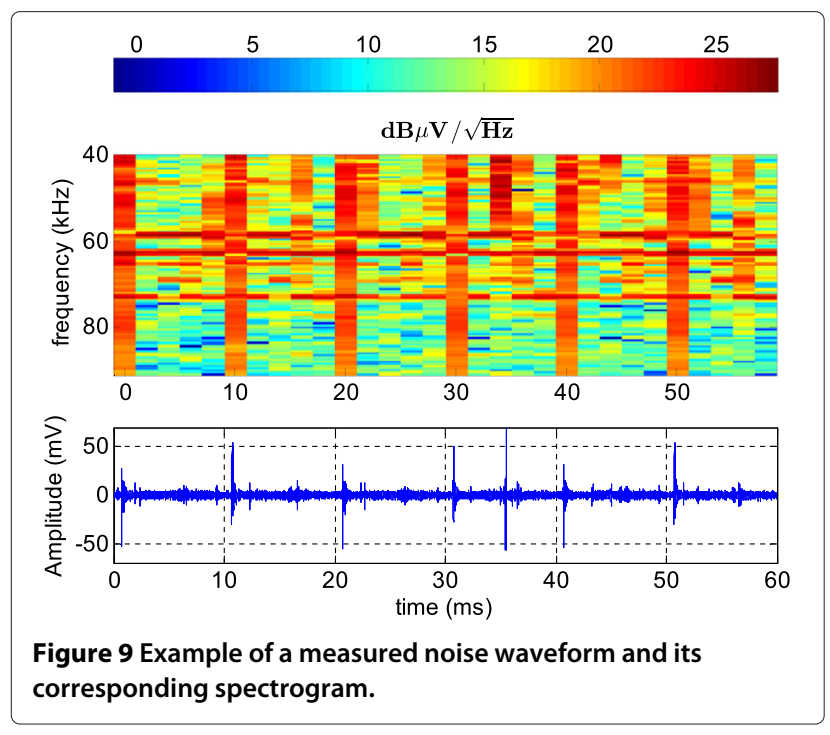

Figure 10 depicts the PSD of the heaviest and the weakest noise register measured in each scenario. The average PSDs have also been included along with their fitting lines obtained using a robust regression,

$$
\operatorname{PSD}(f)=\mathrm{PSD}_{0}-\Delta \mathrm{PSD} \cdot f(\mathrm{kHz}) \quad(\mathrm{dB} \mu V / \sqrt{H z}),
$$

where $\mathrm{PSD}_{0}$ and $\triangle \mathrm{PSD}$ are given in Table 3 . As seen, they have the well-known $1 / f$ decay [15].

Figure 10 also shows the PSD of the 12 noise patterns described in the IEEE P1901.2 model. In this case, the synchronous averaging used for the estimation of the PSD has taken into account that they correspond to a mains frequency of $60 \mathrm{~Hz}$. Their background level is higher than that in the measured ones, especially at high frequencies, where differences can be up to $40 \mathrm{~dB}$. In addition, the modeled noise shows no trace of the NBI. This is somehow surprising because these patterns do clearly reflect $\mathrm{NBI}$ in the frequency range above $100 \mathrm{kHz}$ (not shown in the figure).

In order to characterize the time variation of the noise level, Figure 11 depicts the CDF of the peak and rms variation of the energy along the mains cycle, $E^{j}(\ell)$, computed as described Section 2. Values corresponding to the noise patterns defined in the IEEE P1901.2 have been marked with circles. In this case, the window length has been scaled to take into account that they correspond to a mains frequency of $60 \mathrm{~Hz}$. It can be observed that $50 \%$ of the measured noise registers exhibit peak and rms values higher than 13 and $3.4 \mathrm{~dB}$, respectively. The suitability of the noise model given in the IEEE P1901.2 to reflect the range of the noise level variation can be assessed by its 


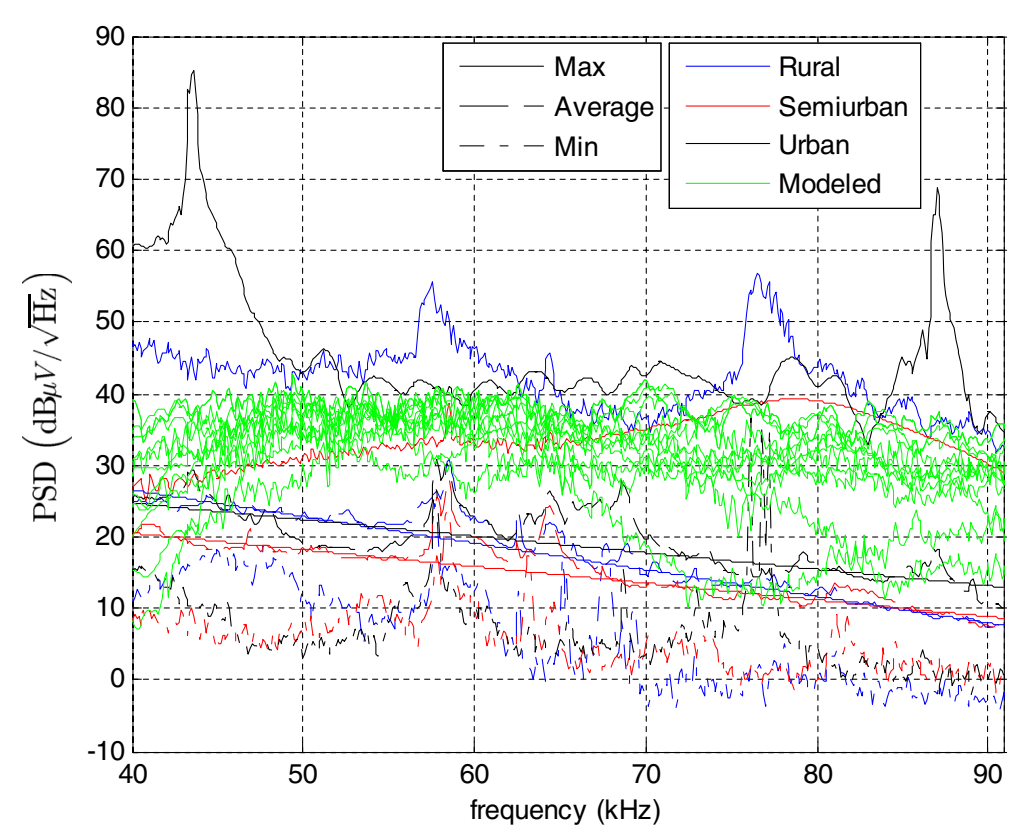

Figure 10 PSDs of the measured and modeled noise.

sampling of the measured curves, which is quite good in almost all the quartiles.

Figure 11 has shown the amplitude of the noise level variation along the mains cycle. However, from a communication system perspective, the rate of this variation is even more interesting. It provides an indication of the data rate gain that could be achieved by using an adaptive modulation strategy [28]. This issue has already been explored in the context of NB-PLC systems in [29]. To this end, the duration of the region in which energy variations are lower than $3 \mathrm{~dB}$ (which would imply a change in the number of bits/symbol when using QAM modulations) has been computed. The CDFs of these values are shown in Figure 12 for the three scenarios, along with the ones of the IEEE P1901.2 noise model. As seen, the rate at which the energy varies in the modeled noise patterns is larger than that in the measured ones. Thus, only one pattern covers $50 \%$ of the measured values. Hence, while the model reflects the magnitude of the measured noise level variations, it underestimates their rate of change. The comparison of the values in Figures 11 and 12 with the FFT window lengths shown in Table 2 confirms that

Table 3 Values of the noise PSD fitting curves

\begin{tabular}{lcc}
\hline Scenario & PSD $_{\mathbf{0}}$ & $\boldsymbol{\Delta}$ PSD \\
\hline Rural & 41.412 & $371.863 \cdot 10^{-3}$ \\
Semiurban & 29.879 & $233.782 \cdot 10^{-3}$ \\
Urban & 33.939 & $230.935 \cdot 10^{-3}$ \\
\hline
\end{tabular}

the use of an adaptive modulation strategy can provide fair data rate gains, as already reported in [29].

\section{Performance assessment}

The most straightforward measure of the performance that can be achieved in a given channel is the capacity. However, it requires precise knowledge of the noise statistics, which are still unknown in NB-PLC. Moreover, simple closed-form expressions are known only for certain distributions, like the Gaussian one. An alternative

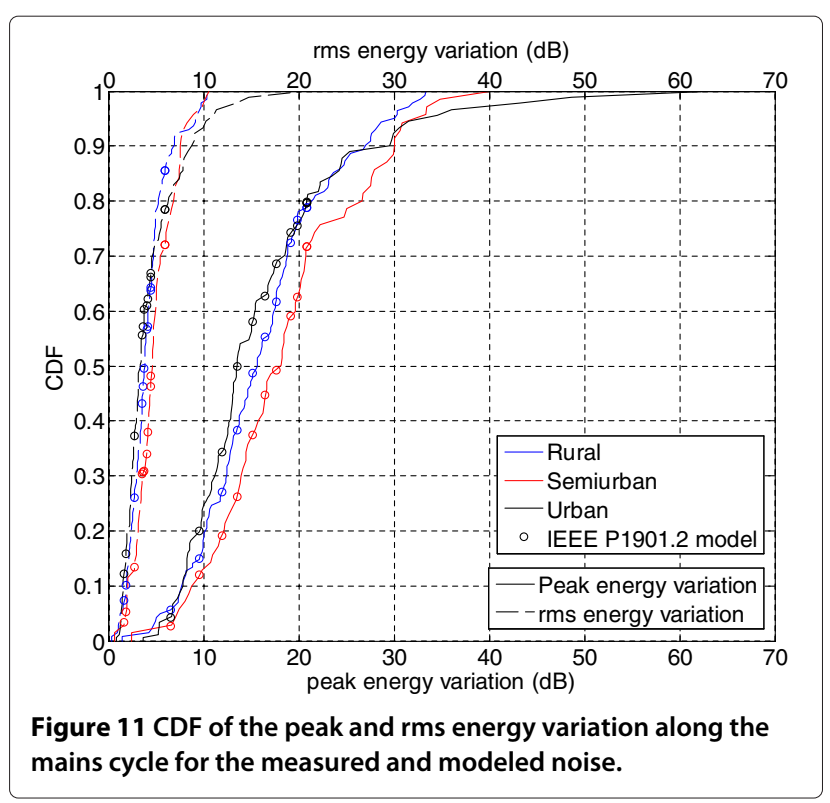




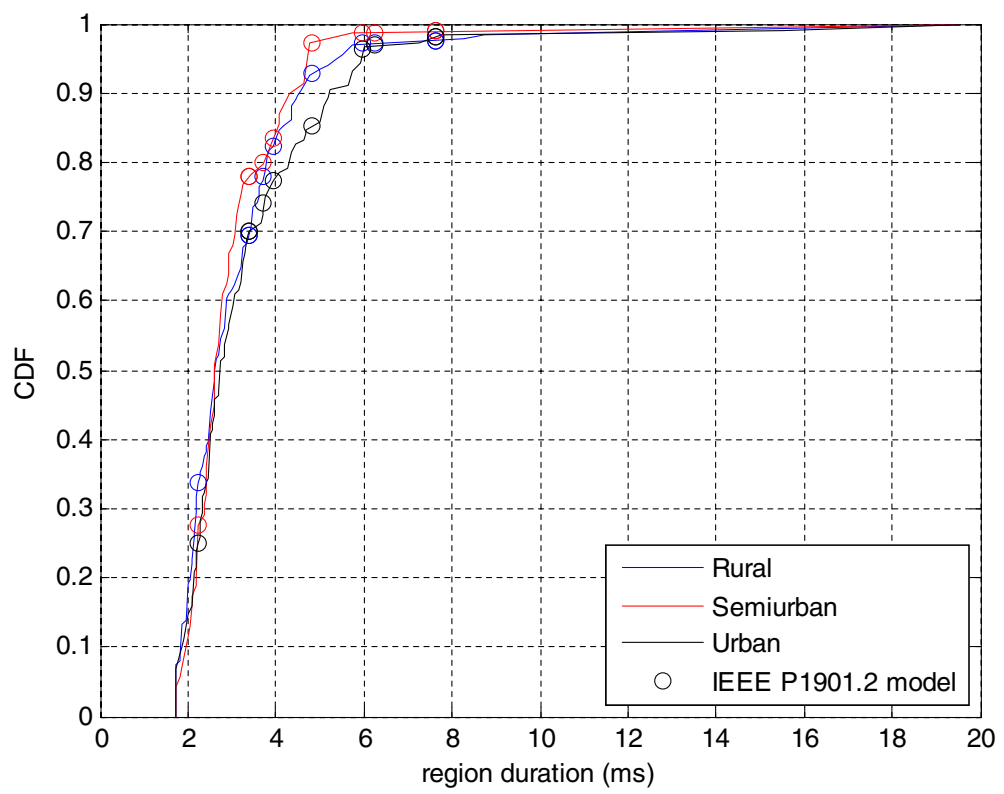

Figure $12 \mathrm{CDF}$ of the duration of the regions in which energy variations are lower than $3 \mathrm{~dB}$.

approach could be to assess the performance that can be attained by standardized systems. Results obtained in this way do not really inform of the potentiality of the channel, which might be under underutilized. Moreover, significant performance differences can be found depending on the algorithms employed at the receiver.

When the noise distribution is unknown, a lower bound on the performance can be obtained by assuming that it is Gaussian-distributed and using the well-known expression of the capacity of a set of parallel flat fading channels with bandwidth $\Delta f$ and stationary Gaussian noise [30]:

$$
R_{\mathrm{SISO}}=\sum_{k \in K} \Delta f \log _{2}\left(1+\frac{P_{k}\left|H_{k}\right|^{2}}{N_{k}}\right),
$$

where $P_{k}, H_{k}$, and $N_{k}$ are the injected power, the frequency response, and the noise power in the $k$ th channel, respectively. $K$ denotes the set of employed frequency indexes. In the case of the MIMO channel, capacity is computed as [30]:

$$
R_{\mathrm{MIMO}}=\sum_{j=1}^{3} \sum_{k \in K} \Delta f \log _{2}\left(1+\frac{P_{k}^{j} \lambda_{k}^{j}}{N_{k}^{j}}\right),
$$

where $j$ denotes the index of the MIMO stream and $\lambda_{k}^{j}, N_{k}^{j}$, and $P_{k}^{j}$ are the eigenvalue, the noise, and the input power at the $k$ th frequency index of the $j$ th stream, respectively.

This is the approach used in this paper. For the sake of simplicity, the values of $N_{k}$ and $N_{k}^{j}$ have been taken from the fitting PSDs whose parameters are shown in Table 3. Noise at the MIMO ports is assumed to be uncorrelated.
However, to provide values as close to the state-of-the-art technology as possible, the following practical constraints have been taken into account: a SNR gap of $5 \mathrm{~dB}$ has been included to model the SNR loss caused by the use of practical constellations; the back-off of the power amplifier at the transmitter has been assumed to be $8 \mathrm{~dB}$, and the maximum number of bits per constellation symbol has been fixed to 6. In order to explore the performance limit of the channel, bit rate values achieved without the latter constraint have also been computed.

Limits for the transmitted level are defined in the EN $50065-1$ [13]. It fixes both the signal level $(134 \mathrm{~dB} \mu \mathrm{V})$ and a PSD mask $(120 \mathrm{~dB} \mu \mathrm{V} / 200 \mathrm{~Hz})$. When using MIMO communications, the maximum level that can be injected on any phase is $6 \mathrm{~dB}$ lower than that in the SISO case. The PSD constraint is the most restrictive one because transmitting at $120 \mathrm{~dB} \mu \mathrm{V} / 200 \mathrm{~Hz}$ in the 40 to $90 \mathrm{kHz}$ band results in a signal level that exceeds $134 \mathrm{~dB} \mu \mathrm{V}$. Hence, in this paper, a flat PSD of $110 \mathrm{~dB} \mu \mathrm{V} / 200 \mathrm{~Hz}$ has been employed.

Figure 13 depicts the relevant part of the CDF of the bit rates attained in the measured SISO and MIMO channels. Curves denoted as 'unconstrained' have been obtained without restricting the number of bits per constellation symbol. Values corresponding to the modeled channels have been computed by combining each of the 300 generated channels whose characteristics have been presented in Section 4 with the 12 noise patterns shown in Section 5. As seen, performance estimated with the models are unrealistically high because of the underestimation of the channel attenuation (see Figure 5), which is larger than 


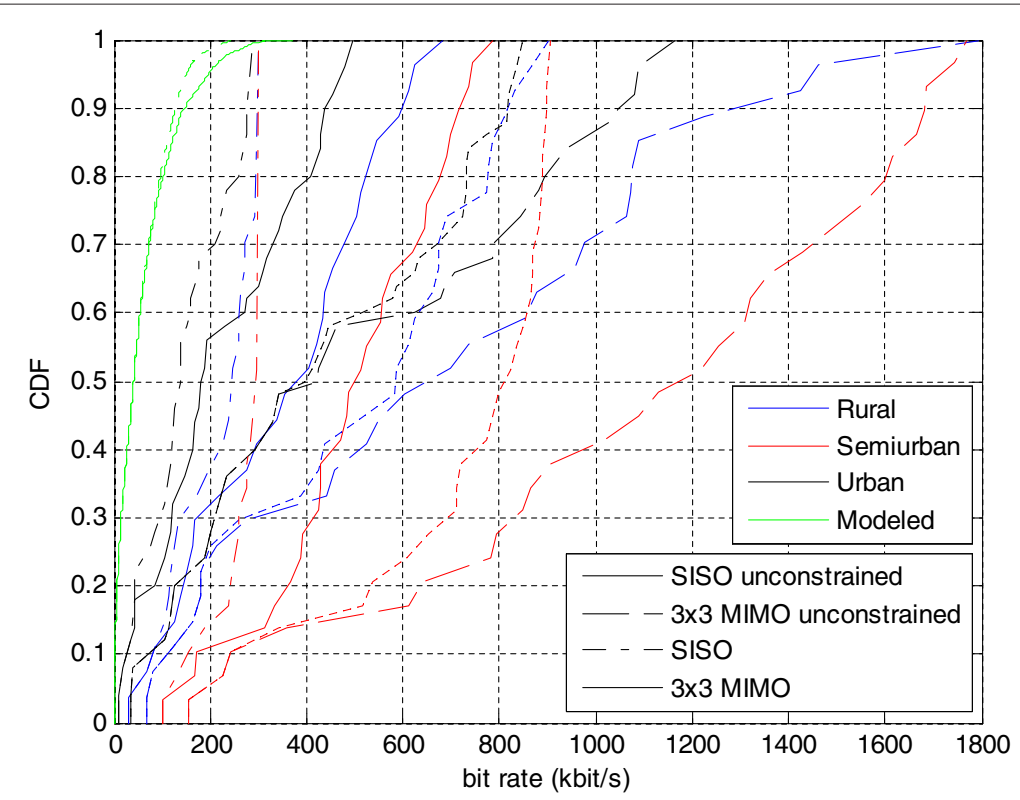

Figure $13 \mathrm{CDF}$ of the bit rate achieved by the MIMO and SISO systems.

the overestimation of the noise levels (see Figure 10). Regarding the measured values, the lower bit rates are obtained in the urban and rural channels. These results are in accordance with the attenuation and noise levels shown in Figures 5 and 10, respectively. They show that the noise level is similar in all scenarios, but rural and urban ones experience higher attenuation. The median bit rates of SISO links in the urban, semiurban, and rural environments are 137, 295, and $246 \mathrm{kbit} / \mathrm{s}$, respectively. It these scenarios, $90 \%$ of the SISO links can deliver more than 28,153 , and $80 \mathrm{kbit} / \mathrm{s}$, respectively.

The comparison of the bit rates in Figure 13 and the ones achieved by the actual systems given in Table 2 reveals that their performance is largely limited by complexity (cost) constraints. In fact, they use constellations with at most 4 bits/symbol. In PRIME, all carriers must employ the same constellation. The same approach has been adopted in G3-PLC, except for the possibility of avoiding transmitting in groups of adjacent carriers with very low SNR. Since the noise in NB-PLC is strongly colored and the channel response is frequency selective, using the same constellation in all employed carriers causes a severe performance degradation. Additionally, to reduce the memory size, the efficiency of the physical layer frames (data symbols length/frame length) has been penalized. In G3-PLC, for instance, the impossibility of fitting more than two Reed-Solomon blocks per frame leads to an efficiency of $78 \%$ when D8PSK is employed.

Figure 13 also shows the bit rate that could be attained with a $3 \times 3$ MIMO system in which the maximum power level allowed by the EN $50065-1$ is injected in all phases. As seen, MIMO can offer significant bit rate improvements when the complexity of the system is constrained. The minimum bit rate attained in $90 \%$ of the channels in the urban, semiurban, and rural environments improves to $73 \mathrm{kbit} / \mathrm{s}(2.61 \times), 241 \mathrm{kbit} / \mathrm{s}(1.57 \times)$, and $108 \mathrm{kbit} / \mathrm{s}(1.45 \times)$, respectively. Performance gains in $50 \%$ of the urban, semiurban, and rural channels are $2.89 \times$, $2.75 \times$, and $2.28 \times$, respectively. It should be taken into account that despite the weak contribution of the third stream, part of the MIMO gain comes from the larger singular values of the first stream with respect to the SISO one. As expected from Figure 8, the lowest gains are obtained in the rural scenario. Removing the constraint on the number of bits per constellation symbol reduces the median values of the MIMO gain in the urban, semiurban, and rural channels to $2.34 \times, 2.35 \times$, and $1.72 \times$, respectively. As expected, it does affect the gains achieved in links with bad transmission characteristics.

\section{Conclusions}

This paper has presented a statistical analysis of the characteristics of NB-PLC channels in the CENELEC$A$ band and has assessed their performance when used for advanced metering infrastructure (AMI) communications in rural, semiurban, and urban scenarios. The accomplished study has included the noise, the channel response, and the input impedance of the power line network. The analysis of the former has examined both its spectral and temporal characteristics. The channel response has been studied in terms of the delay spread, the coherence bandwidth, and the attenuation. An estimation of the data rates that could be achieved has been 
accomplished. The obtained results indicate that the highest performance is achieved in semiurban scenarios and the lowest in urban ones. In the former, $90 \%$ of the channels can deliver more than $153 \mathrm{kbit} / \mathrm{s}$, while in the latter, this figure goes down to $28 \mathrm{kbit} / \mathrm{s}$. Some of the reasons that prevent current NB-PLC systems from achieving these performance have been highlighted. In addition, the use of the three phase conductors for MIMO communications has been explored. It has been shown that a practical $3 \times 3$ MIMO system can give performance improvements larger than $2.61 \times, 1.57 \times$, and $1.45 \times$ in $90 \%$ of the urban, semiurban, and rural channels, respectively.

\section{Endnote}

${ }^{a}$ The low intercarrier spacing in PRIME is motivated by the use of a differential-in-frequency modulation.

\section{Competing interests}

The authors declare that they have no competing interests.

\section{Acknowledgments}

The authors thank Atmel corporation for the support provided to perform all the tests referred in the paper.

The authors would also like to thank the anonymous reviewers for their valuable comments and suggestions.

Received: 6 November 2014 Accepted: 25 February 2015

Published online: 20 March 2015

\section{References}

1. H Farhangi, The path of the Smart Grid. IEEE Power Energy Mag. 8(1), 18-28 (2010)

2. Kema Limited. GB Demand Response. Report 1: The Opportunities for Demand Response. Commissioned by the Energy Association. (Version 1.0) (Kema Limited, Arnhem, 2011)

3. G Strbac, C Kim Gan, M Aunedi, V Stanojevic, P Djapic, J Dejvises, P Mancarella, A Hawkes, D Pudjianto, S Le Vine, J Polak, D Openshaw, S Burns, P West, D Brogden, A Creighton, A Claxton. Benefits of Advanced Smart Metering for Demand Response based Control of Distribution Networks. ENA/SEDG/Imperial College report on Benefits of Advanced Smart Metering (Version 2.0) (Energy Networks Association, London, 2010)

4. H Edelmann, T Kästner. Cost-Benefit Analysis for the Comprehensive Use of Smart Metering. Technical report, Ernst \& Young On Behalf of the Federal Ministry of Economics and Technology (Ernst \& Young GmbH Germany, 2013)

5. S Galli, A Scaglione, Z Wang, For the grid and through the grid: the role of power line communications in the Smart Grid. Proc. IEEE. 99(6), 998-1027 (2011)

6. The OPEN meter Consortium, Description of current state-of-the-art of technology and protocols description of state-of-the-art of PLC-based access technology. European Union Project Deliverable FP7-ICT-2226369, d 2.1 Part 2, Version 2.3, March 2009. http://www.openmeter.com/files/ deliverables/OPEN-Meter\%20WP2\%20D2.1\%20part2\%20v2.3.pdf

7. A Mengi, M Waechter, M Koch, in Proceedings of the IEEE International Symposium on Power Line Communications and Its Applications (ISPLC). $500 \mathrm{kHz}$ G3-PLC access technology for the roll-outs in Germany (Glasgow, 2014)

8. Engage Consulting Limited. High-level smart meter data traffic analysis. Technical report for Energy Networks Association (Energy Networks Association, London, 2010)

9. ITU.T Recommendation G.9902. Narrowband orthogonal frequency division multiplexing power line communication transceivers for ITU-T G.hnem networks (ITU, Geneva, 2012)

10. ITU-T Recommendation G.9903. Narrowband orthogonal frequency division multiplexing power line communication transceivers for G3-PLC networks (ITU, Geneva, 2013)
11. ITU-T Recommendation G.9904. Narrowband orthogonal frequency division multiplexing power line communication transceivers for PRIME networks (ITU, Geneva, 2012)

12. IEEE P1901.2. IEEE Standard for Low-Frequency (less than $500 \mathrm{kHz}$ ) Narrowband Power Line Communications for Smart Grid Applications (IEEE, New York, 2013)

13. European Committee for Electrotechnical Standardization (CENELEC). Signalling on low-voltage electrical installations in the frequency range 3 $\mathrm{kHz}$ to $148,5 \mathrm{kHz}$ - part 1: general requirements, frequency bands and electromagnetic disturbances. European Standard EN 50065-1 (CENELEC, Brussels, 2001)

14. S Galli, Recent developments on the international standardization of narrowband PLC for smart grid applications. Keynote given at the IEEE Internationaly Symposium on Power Line Communications and its Applications (ISPLC) (2012). http://www.ieee-isplc.org/2012/ program_44_1704874382.pdf

15. M Nassar, J Lin, Y Mortazavi, A Dabak, IH Kim, BL Evans, Local utiliy power line communications in the 3-500 khz band: channel impairments, noise, and standards. IEEE Signal Process. Mag. 29(5), 116-127 (2012)

16. M Zimmermann, K Dostert, A multipath model for the powerline channel. IEEE Trans. Commun. 50(4), 553-559 (2002)

17. W Liu, M Sigle, K Dostert, Channel characterization and system verification for narrowband power line communication in Smart Grid applications. IEEE Commun. Mag. 49(12), 28-35 (2011)

18. G Chu, J Li, W Liu, in Proceedings of the, International Symposium on Power Line Communications and Its Applications (ISPLC). Narrow band power line channel characteristics for low voltage access network in China (Johannesburg, 2013)

19. LT Berger, A Schwager, P Pagani, Scheneider DM (eds.), MIMO Power Line Communications: Narrow and Broadband Standards, EMC and Avanced Processing, 1st edn. (CRC Press, Boca Raton, FL, 2014)

20. Y Sugiura, T Yamazato, M Katayama, in Proceedings of the IEEE International Symposium on Power Line Communications and Its Applications. Measurement of narrowband channel characteristics in single-phase three-wire indoor power-line channels (Jeju, 2008), pp. 18-23

21. A Sendin, A Llano, A Arzuaga, I Berganza, in Proceedings of the IEEE International Symposium on Power Line Communications and Its Applications. Strategies for PLC signal injection in electricity distribution grid transformers (Udine, 2011), pp. 346-351

22. AV Oppenheim, RW Schafer, J Buck, Discrete-time Signal Processing. (Prentice Hall, New Jersey, 1999)

23. FJ Cañete, JA Cortés, L Díez, JT Entrambasaguas, Analysis of the cyclic short-term variation of indoor power line channels. IEEE J. Selected Areas Commun. 24(7), 1327-1338 (2006)

24. M Sigle, W Liu, K Dostert, in Proceedings of the, IEEE International Symposium on Power Line Communications and Its Applications. On the impedance of the low-voltage distribution grid at frequencies up to 500 kHz (Beijing, 2012), pp. 30-34

25. A Sendin, I Peña, P Angueira, Strategies for power line communications smart metering network deployment. Energies. 2014(7), 2377-2420 (2014)

26. JG Proakis, Digital Communications. (McGraw-Hill, Massachusetts, 1995)

27. JA Cortés, L Díez, FJ Cañete, JJ Sánchez-Martínez, JT Entrambasaguas, Performance analysis of OFDM modulation on indoor broadband PLC channels. EURASIP J. Adv. Signal Process. 2011(78), 1-12 (2011)

28. A Goldsmith, S Chua, Variable-rate variable-power MQAM for fading channels. IEEE Trans. Commun. 45(10), 1218-1230 (1997)

29. KF Nieman, J Lin, M Nassar, K Waheed, BL Evans, in Proceedings of the International Symposium on Power Line Communications and lts Applications (ISPLC). Cyclic spectral analysis of power line noise in the 3-200 kHz band (Johannesburg, 2013)

30. A Goldsmith, Wireless Communications. (Cambridge University Press, New York, 2005) 